\title{
René Maran à Maurice Barrès : d'un « écrivain français » à l'autre
}

\section{Xavier Luce}

\section{OpenEdition \\ Journals}

Édition électronique

URL : https://journals.openedition.org/coma/7775

DOI : $10.4000 /$ coma. 7775

ISSN : 2275-1742

\section{Éditeur}

Institut des textes \& manuscrits modernes (ITEM)

\section{Référence électronique}

Xavier Luce, "René Maran à Maurice Barrès : d'un « écrivain français » à l'autre », Continents manuscrits [En ligne], 17 | 2021, mis en ligne le 15 octobre 2021, consulté le 08 janvier 2022. URL : http://journals.openedition.org/coma/7775 ; DOI : https://doi.org/10.4000/coma.7775

Ce document a été généré automatiquement le 7 janvier 2022.

\section{(2) $\odot \Theta \Theta$}

Continents manuscrits - Génétique des textes littéraires - Afrique, Caraîbe, dispora est mis à disposition selon les termes de la licence Creative Commons Attribution - Pas d'Utilisation

Commerciale - Pas de Modification 4.0 International. 


\title{
René Maran à Maurice Barrès : d'un « écrivain français » à l'autre
}

\author{
Xavier Luce
}

... grâce à cette faculté d'assimilation qui est

l'apanage de tous ses pareils ${ }^{1} .$.

\section{Introduction}

Le fonds Maurice Barrès de la Bibliothèque nationale de France (NAF 28210) ${ }^{2}$ conserve sept lettres de René Maran adressées à l'auteur du Roman de l'énergie nationale ${ }^{3}$. Leur rédaction s'étale entre 1917 et 1920 . On peut y recueillir de précieuses indications sur l'intention auctoriale ayant guidé Maran dans l'écriture de ses romans et nouvelles. Cette correspondance entre les deux écrivains séparés d'une génération (Barrès est né en 1862, Maran en 1887), quoique brève par rapport aux centaines de lettres échangées avec son cercle d'amis et soutiens (Manoel Gahisto, Phileas Lebesgue, Léon Bocquet, Charles Barrailley, André Lafon), se révèle néanmoins importante par son objet : le sort des indigènes d'Afrique équatoriale française (AEF) et, plus largement, la situation de l'empire colonial français envisagé à l'aune de son ambition civilisationnelle; ainsi que par le choix de son destinataire: Maran s'adresse à un académicien dont l'œuvre jouit d'un grand prestige pour la génération née après la « défaite de Sedan » et la répression de la Commune de Paris et qui est alors engagé comme représentant du peuple français (Barrès est député de Paris depuis 1906 au sein du groupe de l'Entente républicaine démocratique). En outre, les dates entre lesquelles s'échelonnent les lettres de Maran à Barrès correspondent à la période au cours de laquelle a été écrite la célèbre préface de Batouala (entre novembre 1918 et fin 1920). Ces lettres annoncent la préface et lui font ensuite écho de même qu'elles préfigurent l'ethos du héros d'Un Homme pareil aux autres, Jean Veneuse, dont Frantz Fanon se servira comme cas clinique d'aliénation engendrée par la situation coloniale.

2 Dans cet article, je propose d'analyser le discours porté par Maran à son destinataire Barrès en le mettant en perspective avec des éléments postérieurs aux années 
couvertes par la correspondance ; il s'agit en effet d'apprécier le sens de l'engagement d'abord de l'écrivain au sein de la Ligue universelle de défense de la race nègre (LUDRN) en tant que «premier Goncourt noir " puis, à compter des années 1930, du «brillant illustrateur de l'humanisme noir ${ }^{4} "$; en somme, comment Maran concevait-il son «métier d'écrivain français ${ }^{5}$ » et comment celui-ci a-t-il été compris par ses cadets?

\section{Dialectique de l'assimilation}

3 La théorisation par Aimé Césaire de l'assimilation comme dialectique s'avère pertinente pour comprendre la conception que se faisait Maran de son «métier d'écrivain français ${ }^{6} »$. La manière dont il motive l'objet de sa première lettre à Barrès dénote l'emprise du paradigme évolutionniste en fonction duquel il se situe : il se présente comme un exemple de « ce que l'instruction peut faire d'un primitif ${ }^{7}$ ». Cette posture se comprend en fonction de l'idée défendue par l'anthropologue Lewis Henry Morgan selon laquelle « la sauvagerie a précédé la barbarie dans toutes les tribus de l'humanité, comme la barbarie, on le sait, a précédé la civilisation ». Gérard Leclerc - disciple de Georges Balandier, théoricien de la "situation coloniale »-, définit la tâche que se donne «l'anthropologie nouvelle» à la fin du xIx siècle: "[reconnaître] des "coutumes" dont le sens peut être compris à partir de leur mise en relation entre elles, puis avec des ensembles culturels plus vastes qui sont précisément les stades de l'évolution ${ }^{8}$. " C'est ainsi que les fictions africaines de Maran se donnent à lire comme des fables anthropologiques sur les coutumes indigènes dont l'auteur, en tant que fonctionnaire colonial et " nègre évolué », «assimilé », se porte garant de l'authenticité.

4 Cette correspondance avec Barrès permet en ce sens d'appréhender les enjeux de l'assimilation et ses manifestations sur le plan de l'ontologie et du politique chez un "écrivain français " et se revendiquant tel mais dont le phénotype porte, dans l'imaginaire collectif, la marque d'une ascendance primitive et servile associée au continent africain - Maran est né en Martinique de parents afro-guyanais. Elle permet également de mettre en lumière le dialogue critique qui caractérise les «négritudes littéraires ${ }^{9}$ " et qui, aux yeux de la génération de Senghor, fera de René Maran un "précurseur de la négritude ${ }^{10}$ ». Or, avec la mise en place d'une Négritude institutionnalisée, à compter des années 1960, le concept devient «source, peut-être, de nombreux malentendus, mais aussi d'acrobaties périlleuses qui ont transformé le "grand cri nègre" en une clameur "pervertie"11 ". Ce néologisme - négritude - employé par Aimé Césaire en 1935 dans L'Étudiant noir ${ }^{12}$ et repris dans Cahier d'un retour au pays natal ${ }^{13}$, renvoie moins à une idéologie qu'à un « corpus de textes, de discours multiples et parfois contradictoires ${ }^{14}$ ", fait valoir Nadia Yala Kisudiki, à propos, notamment, du colonialisme et du racisme qui lui est intrinsèque. Et ce sont, précisément, "ces reprises, ces précisions et déplacements constants du terme "Négritude" [qui] forment le cœur des questionnements critiques, qu'on retrouve chez les écrivains et penseurs de ce mouvement ». Aussi, "[q]u'elle soit objective, désignant précisément "l'ensemble des valeurs de civilisation du monde noir", ou subjective, renvoyant à une expérience vécue, à une "des formes historiques de la condition faite à l'homme", la "Négritude" est traversée par de multiples généalogies intellectuelles, qui expliquent qu'au-delà des points de convergence qui forment une certaine unité de son concept, ses traductions sur le plan pratique, politique, littéraire et théorique ne se recoupent pas de façon 
nécessaire ${ }^{15}$ ». C'est pourquoi l'intention de Maran et sa mise en scène auctoriale peuvent se comprendre à l'aune, non pas de la Négritude (en tant qu'idéologie politique $\left.{ }^{16}\right)$, mais d'une négritude en tant qu'elle est, en 1910-1920, une "pratique sans théorie ${ }^{17}$ " élaborée en fonction des "questions que pose la présence des Nègres au monde» et de "sa perception par l'Autre et par soi-même ${ }^{18}$ ». C'est dans cette perspective que Matthieu Renault, dans un article intitulé « Amour de la race ou amour au-delà des races ? Frantz Fanon, lecteur de René Maran ", met en lumière ce dialogue lié à une " condition subjective » commune en tant que « Français de couleur » :

Il y a en effet chez Maran des formules qui auraient pu tout aussi bien être celles de Fanon; ainsi de ces lignes de la préface d'Un homme pareil aux autres : "L'Européen en général, le Français en particulier, non contents d'ignorer le nègre de leurs colonies, méconnaissent celui qu'ils ont formé à leur image ». Elles anticipent d'une certaine manière les thèses fanoniennes de l'imposition culturelle, de l'absence de reconnaissance, de l'identification du Noir à l'image du maître ${ }^{19}$.

Le besoin de reconnaissance et la perversion du désir identificatoire sont le produit de la situation d'assimilation dans laquelle est engoncée René Maran qui se présentera, dans le cadre du débat relatif au droit de vote des femmes, comme un des «fils adoptifs » de la France, «fils d'une autre race ${ }^{20}$ ». Cette prise de position en faveur du droit de vote des femmes françaises, par sa motivation en fonction d'une généalogie raciale de la citoyenneté, d'une part, révèle la difficulté de l'écrivain à considérer les femmes afro-descendantes originaires de ces «vieilles colonies» (Guadeloupe, Martinique, Guyane, Réunion) comme sujets politiques et, d'autre part, dénote l'intériorisation du principe républicain assimilationniste.

6 À travers cette correspondance avec Maurice Barrès se lit le drame d'une assimilation contrariée et, pour cause, pétrie de ces contradictions que les écrivains et penseurs rassemblés autour du concept de Négritude viseront à transcender de diverses manières et selon différentes aspirations. De fait, avance Aimé Césaire dans les colonnes de L'Étudiant noir, l'assimilation s'avère un idéal en faux-semblant : « une fois semblable à son formateur, il [le disciple] ne comprend plus le mépris de celui-ci [le maitre] et le hait ${ }^{21} »$. Et de persifler en faisant une allusion voilée aux partisans de l'assimilation, ceux que Buata Malela qualifie d'« assimilationnistes militants ${ }^{22} »:$ « c'est ainsi que j'ai ouï dire que certains disciples haïssent le maître, parce que le maître veut toujours rester le maître, quand le disciple a cessé d'être le disciple ». Il formule ainsi un théorème social assurant que «le colonisateur qui a "assimilé" se dégoûte vite de son œuvre ${ }^{23}$ ». Une vingtaine d'années plus tard, au Congrès des écrivains et artistes noirs, Césaire citera le nom de Maran avec celui de Jean-Joseph Rabearivelo pour exemplifier, cette fois-ci, sa théorisation de l'assimilation comme dialectique :

[...] toute vraie création artistique indigène, le colonisateur ne peut la regarder que d'un œil soupçonneux. Il peut essayer de s'en accommoder. Il peut même essayer de l'utiliser. Mais au fond toute création indigène, pour le colonisateur est insolite, donc dangereuse. Si on en voulait, parmi tant d'autres, une preuve, il suffirait de se rappeler les débuts de la littérature nègre en France et la situation scandaleusement hostile faite il y a trente ans à un René Maran ou à un Rabearivelo... Par leur seule existence ils constituaient un scandale... Et dans la même mesure où elle est dangereuse pour le colonisateur, elle est rassurante au sens propre du mot pour le colonisé, je veux dire qu'elle fait contrepoids au complexe d'infériorité dont c'est la mission de toute colonisation que de l'instiller au colonisé ${ }^{24}$. 
7 En appréhendant l'assimilation comme une dialectique, c'est-à-dire en fonction d'un mouvement socio-historique amené à évoluer d'une génération à l'autre et d'un événement à l'autre, Césaire établit un paradigme critique pour comprendre le positionnement auctorial de Maran et sa portée dans le contexte qui était le sien. Gisèle Sapiro, dans son essai Peut-on dissocier l'œuvre de l'auteur ? fait valoir l'importance de la contextualisation, sans quoi l'« [o]n observe alors une logique d'euphémisation visant à dépolitiser [l'œuvre] [...] en la détachant de ses conditions initiales de production et de publication, c'est-à-dire de la constellation des significations dans laquelle elle s'inscrivait à l'origine ${ }^{25}$ ». C'est pourquoi les stratégies discursives mises en œuvre par Maran nous deviennent intelligibles à travers l'étude des discours critiques suscités chez ses contemporains, notamment chez ses cadets et sans doute davantage chez ceux ayant une expérience du racisme en colonie ; et sans doute davantage encore chez ceux ayant procédé à une tentative de compréhension de « l'arsenal complexuel qui a germé au sein de la situation coloniale ${ }^{26}$ » selon les termes de Frantz Fanon, né quelques années après le Goncourt 1921. C'est le cas d'André Fraisse: né en 1909, il est administrateur en Indochine puis en Afrique. À la fin de sa carrière, il se lance dans l'écriture d'un "mémoire de décolonisation » dans lequel il procède à un examen de conscience en essayant de poser un regard lucide sur l'entreprise coloniale elle-même sans toutefois remettre en question le paradigme civilisationnel dans lequel lui et Maran ont été formés, à savoir l'anthropologie évolutionniste ${ }^{27}$. Au cours de son gouvernorat en Oubangui-Chari dans les années 1950, Fraisse rassemble un certain nombre de pièces administratives à présent consultables aux archives nationales d'Outre-mer qui offrent un aperçu de cette "vie coloniale » et, particulièrement, de celle qu'aura vécue René Maran. Un dossier intitulé «Le conflit du blanc et du noir » contient des papiers de l'écrivain et, parmi ceux-ci, une lettre datée du 24 avril 1952 dans laquelle ce dernier déplore la faible réception d'Un homme pareil aux autres :

Tôt ou tard, on discutera de mes idées. On sera alors forcé de se rendre compte qu'elles ont toujours été la vérité même [je souligne], et qu'il m'a fallu du courage pour consacrer ma vie à les exposer avec le plus parfait désintéressement, et en toute tranquillité d'esprit ${ }^{28}$.

8 C'est le cas aussi de Léopold Sédar Senghor, de Léon Gontran Damas et d'Aimé Césaire nés respectivement en 1906, 1912 et 1913. De même que la génération de Bernard de Chartres avait conscience d'être " assis[e] sur des épaules de géants ", celle qui émerge à compter des années 1930 autour de L'Étudiant noir voit en Maran un de ces géants à partir duquel le problème colonial et celui du racisme ont pu être posés et pensés de manière radicale. En effet, fera observer Césaire à la tribune de l'Assemblée nationale où il travaille de concert avec Léopold Sédar Senghor et leur aîné Maran qui se positionne comme « le plus modéré des trois ${ }^{29}$ »-, la décolonisation et l'indépendance se tiennent en germe dans la promesse non tenue de l'assimilation: "Quand nous voulons nous assimiler, nous intégrer, vous nous rejetez, vous nous repoussez. Quand les populations coloniales se libèrent, vous les mitraillez ${ }^{30}$ !» Avant lui, Maran mettait en garde :

Il faut que de toute urgence la Chambre actuelle et le gouvernement accordent aux indigènes des colonies les droits élémentaires qu'ils réclament. Sinon les sirènes auront beau temps pour chanter la révolte ${ }^{31}$.

Lorsque Maran écrit à Barrès, il a conscience que l'image de la France offerte aux yeux des populations administrées comporte un enjeu géopolitique de premier ordre. Aussi est-il significatif que, dans une de ses lettres, il mentionne parmi ses lectures le Prix 
Nobel de littérature en 1916 Rabindranath Tagore ${ }^{32}$ : dans la préface de Batouala, il fera écho à la conférence prononcée par ce dernier à l'Université impériale de Tokyo le 18 juin 1916 pour évoquer ces « graves questions que la civilisation occidentale a posées devant le monde ${ }^{33}$ " qui sont, à ses yeux, celles du racisme et de la mission civilisatrice $^{34}$. Ayant été témoin dans le cadre de ses fonctions d'agent colonial en AEF des «malversations" et des "atrocités qui y abondent ${ }^{35}$ ", les discours officiels lui apparaissent comme de vains mots, criminels en ce que ceux-ci masquent une abominable réalité. C'est l'objet principal de sa correspondance avec Barrès auquel il relate cette « vie coloniale » qui décivilise :

Nous sommes, ici, plusieurs à étouffer sous une honte inavouable. Déjà, en temps de paix, les travaux que nous faisions étaient rien moins que propres. Sous prétexte de civiliser des peuplades énervées, nous les avions surchargées de prestations de toutes sortes, nous les avions asservies. En échange, de nous elles ne recevaient que du dédain, du mépris et des coups. Cela se sait dans les milieux coloniaux. On se garde de le dire ${ }^{36} .$.

[...] nous, les fonctionnaires, nous ne sommes pas venus ici pour être des négriers. Nous en avons assez de razzier ces malheureux, tirailleurs volontaires qu'on attache pour qu'ils ne se sauvent pas. Nous en avons trop fait déjà, trop vu. Qu'on ne nous oblige pas à salir nos mains davantage! La France ne peut pas nous demander cela! Ce n'est pas vrai! Ce n'est pas vrai ${ }^{37}$ !...

Je voudrais être calme. Comment l'être! Lorsque je suis entré dans l'administration coloniale, j'étais loin de penser que j'allais devenir une manière de bandit. Je ne suis pourtant pas autre chose $\mathrm{e}^{38}$. 
Fig. 1 : Caricature jointe à la lettre du 28 février 1918

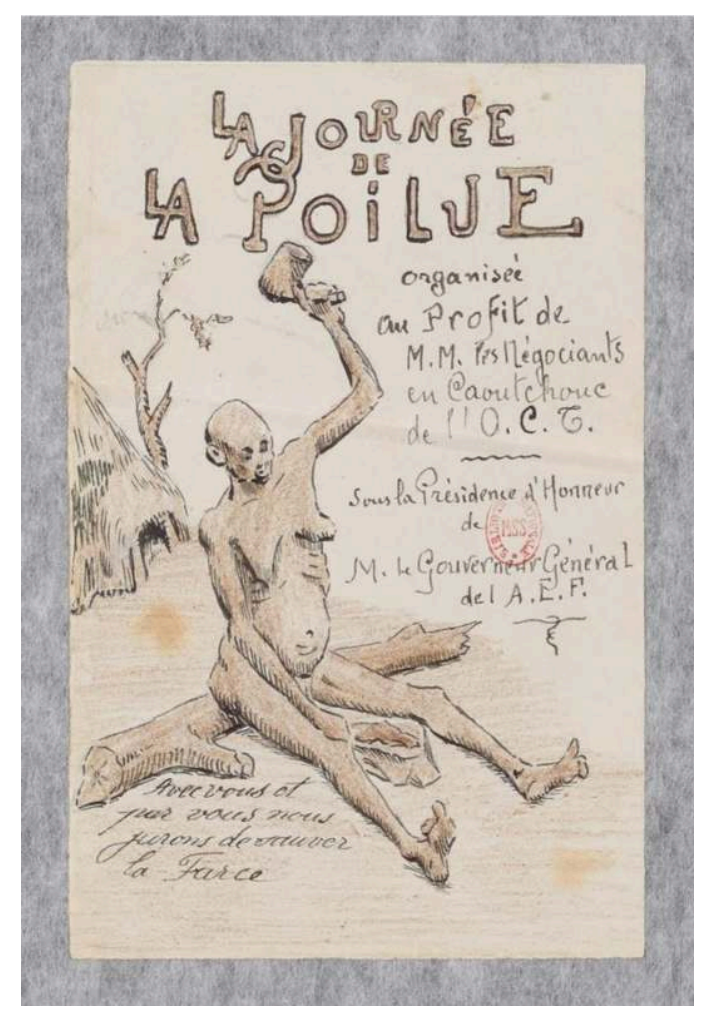

Dans cette lettre Maran écrit : « Je vous disais, plus haut, que nous étions plusieurs, ici, à étouffer sous une honte inavouable. L'un de nous, un français, un breton, a fait cette caricature que vous avez trouvée avec ma lettre. Elle est amère. Elle est terrible. Société des Nations ! Quand donc le byzantisme laissera-t-il, pour d'autres ciels, la terre de France ? Société des Nations ? Quelle ironie ! On n'est pas encore parvenu au respect de l'individu. Société des Nations !... »

BnF, fonds Maurice Barrès (NAF 28210)

Et c'est là encore tout l'objet de son roman Batouala tel qu'il l'expose dans sa préface : révéler l'écart entre les discours lénifiants et les opérations sur le terrain.

Civilisation, civilisation, orgueil des Européens, et leur charnier d'innocents, Rabindranath Tagore, le poète hindou, un jour, à Tokio, a dit ce que tu étais !

Tu bâtis ton royaume sur des cadavres. Quoi que tu veuilles, quoi que tu fasses, tu te meus dans le mensonge. A ta vue les larmes de sourdre, et la douleur de crier. Tu es la force qui prime le droit. Tu n'es pas un flambeau mais un incendie. Tout ce à quoi tu touches, tu le consumes...

Honneur du pays qui m'a tout donné, mes frères de France, écrivains de tous les partis; vous qui, souvent, disputez d'un rien, et vous déchirez à plaisir, et vous réconciliez tout à coup, chaque fois qu'il s'agit de combattre pour une idée juste et noble, je vous appelle au secours, car j'ai foi en votre générosité39.

Ce célèbre passage de sa préface pourrait avoir été écrit dès novembre 1918 à en croire son article intitulé «Ma condamnation » paru dans le dernier numéro des Continents, organe de la Ligue universelle de défense de la race nègre (LUDRN): "c'est pour déclencher une enquête que j'ai écrit la préface de Batouala ${ }^{40} »$. C'est ce que répètera Maran à André Fraisse, une trentaine d'années plus tard ${ }^{41}$. Sa lettre du 28 février 1918 tend à le confirmer puisqu'on y trouve, en latence, le «terrible couplet $^{42}$ " sur la civilisation si abondamment reproduit jusqu'à aujourd'hui :

La civilisation n'a apporté que la mort. Où qu'elle passe, la destruction au squelette sordide apparait. C'est ce que disait l'an dernier à Tokyo, le très remarquable poète 
Rabindranath Tagore. Et vous savez bien que le grand intellectuel hindou a raison ${ }^{43} \ldots$ moment où il annonce avoir achevé, «depuis quatre ans », soit donc en 1913, "un roman sur les mœurs indigènes» dans lequel il «relate leurs faits, leurs gestes, leurs propos", ces derniers se révélant parfois «troublants", indique-t-il avec laconisme. Nous savons, notamment par sa correspondance avec son éditeur Léon Bocquet, que ce roman est Batouala - bien qu'il travaille, en parallèle, sur plusieurs projets à la fois dont la nouvelle $D_{j o g o n i}{ }^{44}$ et un récit qui s'intitule alors Une amie ${ }^{45}$, "petit roman psychologique", comme il le présente, "dont le héros, à certains moments, [lui] ressemble à s'y méprendre ${ }^{46} »$.

\section{Un homme à nul autre pareil} d'Oubangui-Chari où René Maran est en poste : il interpelle Maurice Barrès sur le sort réservé aux indigènes d'Afrique en cette année charnière où l'on envisage de faire appel à une « force noire » pour soutenir l'effort de guerre.

Je ne vous écris que pour plaider une cause - parce que je suis nègre. En effet, pour moi, l'une des tristesses de cette guerre est que de plus en plus l'on considère mes frères en couleur comme des mercenaires, non comme des êtres perfectibles. Et pourtant... ${ }^{47}$

Fig. 2 : Les deux premiers feuillets de la lettre à Maurice Barrès du 12 janvier 1917
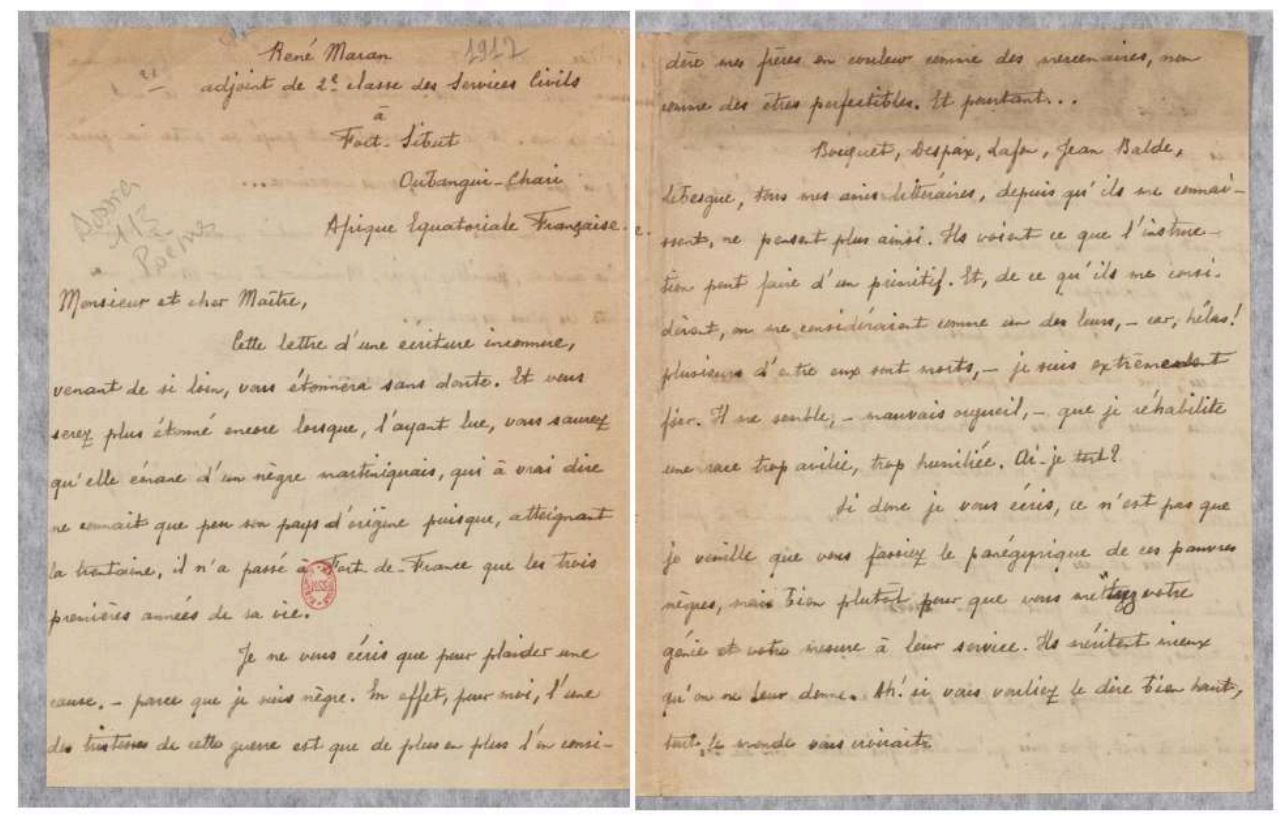

BnF, fonds Maurice Barrès (NAF 28210) 
Fig. 3 : Feuillets 3 et 4 de la lettre du 12 janvier 1917
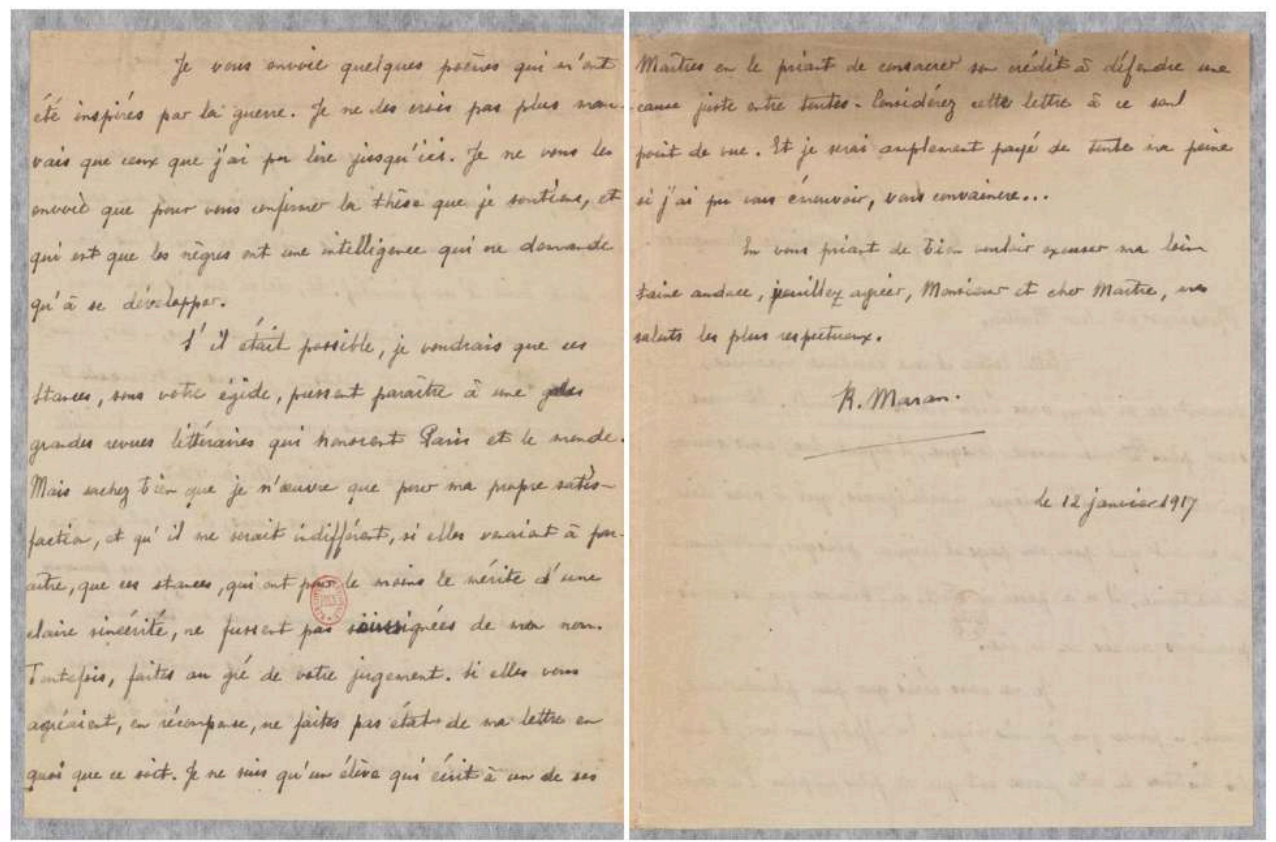

BnF, fonds Maurice Barrès (NAF 28210)

14 Afin de retenir l'attention de son «cher Maître » et de s'en attirer les bonnes grâces, il joint à sa première lettre « quelques poèmes qui [lui] ont été inspirés par la guerre ${ }^{48}$ ».

\section{Fig. 4 : Tapuscrit joint à la lettre du 12 janvier 1917}

II s'agit du poème «ô enchanteurs de l'esprit » faisant partie d'un ensemble de stances, « Vers la sagesse », qui deviendra « Sous la dictée de Marc Aurèle ». 


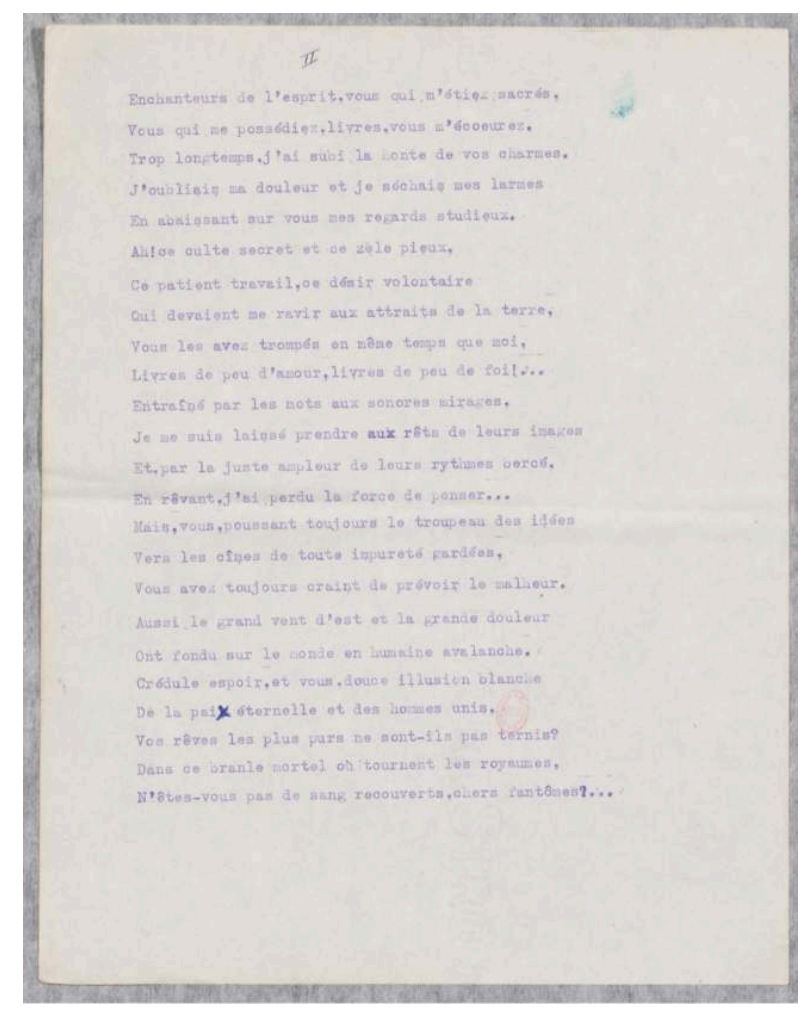

BnF, fonds Maurice Barrès (NAF 28210)

S'il était possible, je voudrais que ces stances, sous votre égide, pussent paraître à une des grandes revues littéraires qui honorent Paris et le monde. Mais sachez bien que je n'œuvre que pour ma propre satisfaction, et qu'il me serait indifférent, si elles venaient à paraître, que ces stances, qui ont pour le moins le mérite d'une claire sincérité, ne fussent pas signées de mon nom. Toutefois, faites au gré de votre jugement. Si elles vous agréaient, en récompense, ne faites pas état de ma lettre en quoi que ce soit. Je ne suis qu'un élève qui écrit à un de ses Maîtres en le priant de consacrer son crédit à défendre une cause juste entre toutes. Considérez cette lettre à ce seul point de vue. Et je serai amplement payé de toute ma peine si j'ai pu vous émouvoir, vous convaincre... ${ }^{49}$

Dans sa lettre suivante, de Fort-Crampel en date du 13 juillet 1917, il révèle la raison de cette pièce jointe :

Si donc je vous ai envoyé ces poèmes, qui ne sont pas encore au point, c'est que je voulais, par eux, vous expliquer ma lettre. Je voulais vous prouver que je pouvais me permettre de vous l'écrire. Que ma culture même me poussait à cette audace. Et que c'était lâcheté, que de ne vous dire pas ce que je voyais, ici ${ }^{50} .$.

La correspondance qu'initie René Maran avec Maurice Barrès s'inscrit d'emblée sous le signe d'un engagement à dire, entendu que dire c'est exposer au grand jour ce qui se sait au sein des milieux coloniaux - ce qu'il souligne dans la préface de Batouala ${ }^{51}$ - mais que l'on dissimule aux yeux de l'opinion publique, cette « opinion publique ${ }^{52}$ » que le héros d'Un homme pareil aux autres, Jean Veneuse, visera à mettre au fait.

Dans sa lettre du 8 décembre 1918, cette passion pour la vérité et sa révélation s'exprime à travers une réticence - rhétorique ? - à raconter ce qui serait de l'ordre du trop bien connu de la part des intéressés (les hauts fonctionnaires et le ministre des Colonies) mais ignoré de ses « frères en esprit, écrivains de France » qu'il exhorte, dans la préface de son premier roman publié, à endosser leur responsabilité en tant que «mainteneurs [de] [...] la nation ${ }^{53} »$. 
Tout le monde sait qu'en général les fonctionnaires coloniaux français sont des déchets de civilisation, de morale et d'intelligence. Au surplus, vous ne pouvez savoir combien ils haïssent le nègre, que ce nègre soit l'indigène où [sic] qu'il soit un «produit français", ayant des diplômes, marié ou fiancé en France à une française. Sous toutes ses apparences, le nègre c'est l'ennemi ${ }^{54}$.

Dans ces lettres, l'écrivain confie ce qu'il a sur le cœur. Car, bien évidemment, ce nègre «produit français » est Maran lui-même. Dans sa première lettre, il se présente comme « nègre martiniquais » mais, tient-il à préciser, « qui à vrai dire ne connait que peu son pays d'origine puisque, atteignant la trentaine, il n'a passé à Fort-de-France que les trois premières années de sa vie ${ }^{55} »$. Et de renchérir dans la lettre suivante en mettant l'accent sur son " patriotisme intact» :

Car je suis français, - français de cœur et d'esprit. Passionné de la France, et du génie de sa langue, je ne pouvais pas ne pas être patriote. Je suis né à la Martinique. Mais c'est Bordeaux qui est ma ville natale, c'est la France qui est mon pays. J'y ai toujours vécu, et chaque congé me ramène à lui...56

19 Ce même ethos qui suscite l'amour de l'écrivain pour la France est la cause de son rejet haineux en tant qu'il est d'abord perçu comme "nègre ». Par l'usage de la troisième personne - « que ce nègre [...] soit un "produit français", ayant des diplômes, marié ou fiancé en France à une française ${ }^{57} »-$, Maran semble se regarder depuis un poste d'observation extérieur à sa corporéité. Cette posture anticipe l'expérience de dépersonnalisation relatée par celui qu'on peut, avec Frantz Fanon, s'autoriser à considérer comme son avatar, Jean Veneuse: «Je suis là, dans cette foule, moi, Jean Veneuse. J'assiste à mon propre départ. Je me suis dédoublé, dépersonnalisée ${ }^{58}$. " Toute l'intrigue de ce roman sentimental, telle que l'ont analysée Tina Harpin et Laura Gauthier Biasi ${ }^{59}$, repose sur la poursuite d'un amour idéel à l'endroit d'une femme blanche, une "française » écrit-il par litote - c'est que l'emploi de l'adjectif «français » sous-entend celui de blanc en fonction du contexte ${ }^{60}$.

Dans un article intitulé « Dépersonnalisation, le doute d'exister? », le psychiatre Didier Lauru fait ressortir les enjeux existentiels de cette expérience : le sujet « ressent [...] douloureusement un écart entre lui et la conscience de son existence, confinant à un sentiment extrême d'incomplétude du sujet». Pour Lacan, "la dépersonnalisation commence avec la non-reconnaissance de l'image spéculaire ${ }^{61} »$. Il semble que René Maran conçoive sa fonction auctoriale sur le mode d'un dédoublement lié à une expérience de dépersonnalisation. Dans la préface de Batouala, cette dissociation apparaît en creux lorsqu'il fait état de "cahiers " qu'il conserve, pour l'heure, par devers lui et qu'il distingue du roman publié :

Et, plus tard, lorsqu'on aura nettoyé les suburres coloniales, je vous peindrai quelques-uns de ces types que j'ai déjà croqués, mais que je conserve, un temps encore, en mes cahiers. Je vous dirai qu'en certaines régions, de malheureux nègres ont été obligés de vendre leurs femmes à un prix variant de vingt-cinq à soixantequinze francs pièce ${ }^{62}$.

Puis, au moyen d'une épanorthose, il en précise la modalité :

Je vous dirai... Mais, alors, je parlerai en mon nom et non pas au nom d'un autre; ce seront mes idées que j'exposerai et non pas celles d'un autre ${ }^{63}$.

Ces «cahiers" contiendraient ses propres «idées» et non celle de ces "pauvres nègres ${ }^{64} »$ dont, en tant qu'administrateur auteur d'un roman colonial, il donne à lire voire, à entendre, sur le mode d'un « roman parlant ${ }^{65}$ »- leurs " propos » susceptibles de « [troubler] » la conscience métropolitaine. 
Cette dépersonnalisation vécue par Maran se comprend à l'aune du racisme dont il relate, pudiquement, les manifestations au sein de l'administration coloniale. C'est que " nègre " parmi " les nègres", comme il le fera dire par Jean Veneuse dans une lettre adressée à son « Andrée chérie », cette Française blanche, il n'est pas vraiment nègre :

D'abord, je ne suis pas noir, ce qu'on appelle noir. Ensuite, je ne suis pas un noir comme les autres. [...] Venu à Bordeaux tout enfant, à une époque où il aurait été difficile d'y trouver huit ou dix nègres, mes meilleurs amis sont des blancs. Je pense et vis à la française. La France est ma religion. Je ramène tout à elle. Enfin, hormis ma couleur, je me sais Européen. Je ne peux, en conséquence, que me marier avec une Européenne ${ }^{66}$.

C'est la signification viscérale du titre du roman: un homme similaire aux autres Français, blancs de peau, et, donc, différents des autres «nègres » avec lesquels il ne partage que la couleur de peau. Cet autre que soi représente moins l'altérité que la double identité vécue par Maran alias Veneuse, identité scindée par son phénotype qui signale, aux autres, une présence singulière au monde. Or, de cette singularité, le sujet assimilé n'a que faire ; il se veut, foncièrement, tout comme. Le titre du roman contient ainsi tout le drame de l'assimilation, cette expérience d'un tiraillement ontologique conduisant à l'aliénation de soi. C'est que le sujet nègre assimilé définit son Moi par la médiation d'un sujet-étalon, ce Blanc, en tant que fantasmagorie sociale; en cela, dit Fanon, le sujet-assimilé est « un crucifié67 ».

Cette fois, à la première personne, Maran poursuit son récit épistolaire en abordant sa propre expérience et celle de son père :

Il y a neuf ans que cette vérité m'est apparue évidente [la haine du nègre]. Mon pauvre père, lui, en est mort de chagrin, après vingt ans de service durant lesquels il fit, sans jamais pouvoir être titulaire dans ses fonctions, des intérims très distingués de secrétaire général et de gouverneur, et au cours desquels il fut proposé, sans pouvoir l'obtenir jamais, trois fois pour la légion d'honneur. Je suis donc fixé depuis longtemps sur le sort qui m'est réservé. Un nègre, quel qu'il soit, où qu'il soit parvenu sur l'échelle de l'intelligence, ne peut prétendre qu'à des emplois subalternes, - ceux de premier rang ne devant, sans aucun prétexte, lui être attribués ${ }^{68} .$.

Maurice Barrès apparaît ainsi comme le parangon de ces " écrivains de tous les partis " qu'il «appelle au secours, car », écrit-il dans la préface de Batouala, «j'ai foi en votre générosité69. » À son « lointain » et « bienveillant » correspondant, il motive le sens de sa démarche :

Vous voyez, à présent, pourquoi je vous ai écrit! Une immense gloire s'attache à votre nom, à vos œuvres, à votre vie politique, la seule qui nous soit connue. Je voudrais, nous voudrions vous voir vous dresser entre nous et cette ignominie ${ }^{70}$.

Cette « ignominie », c'est l'abandon des colonies à ces « déchets de civilisation ${ }^{71}$ » dont souffre Maran d'autant qu'il est désormais question de transformer les « indigènes » en «mercenaires» au service de la patrie en guerre. Or, fait-il observer, «bien que la France, ou ceux qui la représentent, n'aient rien fait pour ses colonies, - depuis qu'elle est en guerre, elle leur demande tout ». Voilà qui est inconcevable : « La France ne peut pas nous demander cela! Ce n'est pas vrai! Ce n'est pas vrai ${ }^{72} ! .$. » écrit Maran sur le coup d'une émotion qu'il peine à contenir.

Je ne vous explique que très imparfaitement tout ce que je ressens. Je voudrais vous inciter à attaquer cette légende que l'on forme sur l'inépuisable richesse humaine de nos colonies d'Afrique. On n'envoie pas des moutons à l'assaut des tranchées boches? Alors qu'on laisse chez eux ces involontaires, qui ne comprennent pas, et 
qui ne savent qu'une chose : c'est qu'après les avoir accablés d'impôts, de taxes et de vexations, on veut les envoyer à la tuerie comme à un châtiment ${ }^{73}$... l'action se veut inébranlable, que rien ne saurait dévier de la vertu. Celle-ci se donne à lire comme la garantie de la véracité des faits allégués: «Je ne suis curieux que d'être vrai » proclame-t-il en insistant sur le fait qu'il se garde bien de "mettre de la "littérature" là où il n'en faut pas ", ceci n'étant pas «un de [ses] procédés de rhétorique $^{78}$ ». En effet, l'engagement maranien se prétend non partisan, n'obéissant qu'au seul souci de "faire éclater [une] vérité 79 », soit la « thèse " que l'écrivain défend et incarne tout à la fois: "les nègres ont une intelligence qui ne demande qu'à se développer ${ }^{80}$.»

cest au sein d'un "nous » thuriféraire de la Civilisation, française en particulier, que Maran s'exprime, mais dont il se distingue par sa généalogie raciale. Ce tiraillement apparaît nettement dans ses lettres à Barrès et permet d'apprécier le sens de son engagement au sein de la LURDN quand il objurgue les autorités françaises à prendre la mesure du problème colonial :

Plus de discours, plus de formules imagées, des actes et vite si l'on veut sauver des races en péril de mort, des races dont on a besoin, si l'on veut renouer avec les Grandes Traditions de la France des Droits de l'Homme et du Citoyen et travailler pour l'humanitées.

La véhémence du discours maranien s'inscrit dans le mouvement abolitionniste incarné par Victor Schœlcher et poursuivi, après la conférence de Berlin (1884-1885), par Benito Sylvain dénonçant, dans sa thèse soutenue à la faculté de Droit de l'Université de Paris, les « succédanés à peine moins odieux ${ }^{82}$ » de l'esclavage institué dans les colonies européennes. De fait, écrira Albert Londres dans Terre d'ébène, éloquemment sous-titré 
La traite des Noirs, "L'esclavage en Afrique n'est aboli que dans les déclarations ministérielles de l'Europe ${ }^{83}$ ". D'où la signature, le 25 septembre 1926, à Genève, de la Convention relative à l'esclavage proclamant «nécessaire d'empêcher que le travail forcé n'amène des conditions analogues à celles de l'esclavage »...

\section{Un souci de perfectibilité}

Un souci de perfection scande ces lettres adressées à Barrès. Dans sa lettre du 13 août 1917, il fait montre d'un zèle perfectionniste :

Je n'avais pas fait la toilette de mes poèmes. Certains étaient parfaits. Je veux dire par là que je les avais amenés jusqu'à ce point qui, actuellement, pour moi, constitue la perfection.

Mais, pour quelques stances parfaites, combien d'autres ne valaient rien! Les unes et les autres, je les ai reprises mot à mot, phrase à phrase, rythme à rythme, car je suis de goût difficile et d'oreille délicate. Je les ai expurgées autant que faire se pouvait. Je vois que, maintenant, elles vous plaisent. C'est du moins mon souhait le plus secret ${ }^{84}$.

34 Maurice Barrès, qu'il considère comme "un des Maîtres de la pensée française ${ }^{85}$ ", apparaît à la fois comme un étalon de référence et un juge. Dans un pli remis par le député de la Guadeloupe Gratien Candace à son homologue Barrès en date du 29 mai 1920 , il sollicite un « appui » littéraire :

Je vous ai déjà dit, et vous savez que je suis nègre. Je voudrais que l'on prouvât à ceux qui feignent de ne pas l'admettre que les nègres sont des hommes comme tout le monde. Vous pourriez, en me donnant votre voix et votre appui, sur lesquels je compte, faire éclater la vérité de ce que j'avance. Je me pose en défenseur d'une race qu'on a trop souvent avilie et que l'on abaisse sans motif, - race qui a cependant sacrifié bien de ses fils à la France et à la cause qu'elle défendait: la Civilisation.

Vous m'aviez promis votre bienveillance, il y a deux ans, et soutenu. Vous ne pouviez savoir l'émotion et la gratitude que je vous devrais si vous me donniez votre voix, - qui consacrerait le talent d'un écrivain modeste, laborieux et... nègre ${ }^{86}$.

La phrase que souligne René Maran contient à la fois la thèse et, à quelques mots près, le titre du roman Un homme pareil aux autres. Dans sa quatrième lettre, il envoie deux portraits de lui car, explique-t-il, « on aime à connaître le visage des gens auxquels on s'intéresse ${ }^{87} »$. 
Fig. 5 : Lettre du 28 octobre 1917

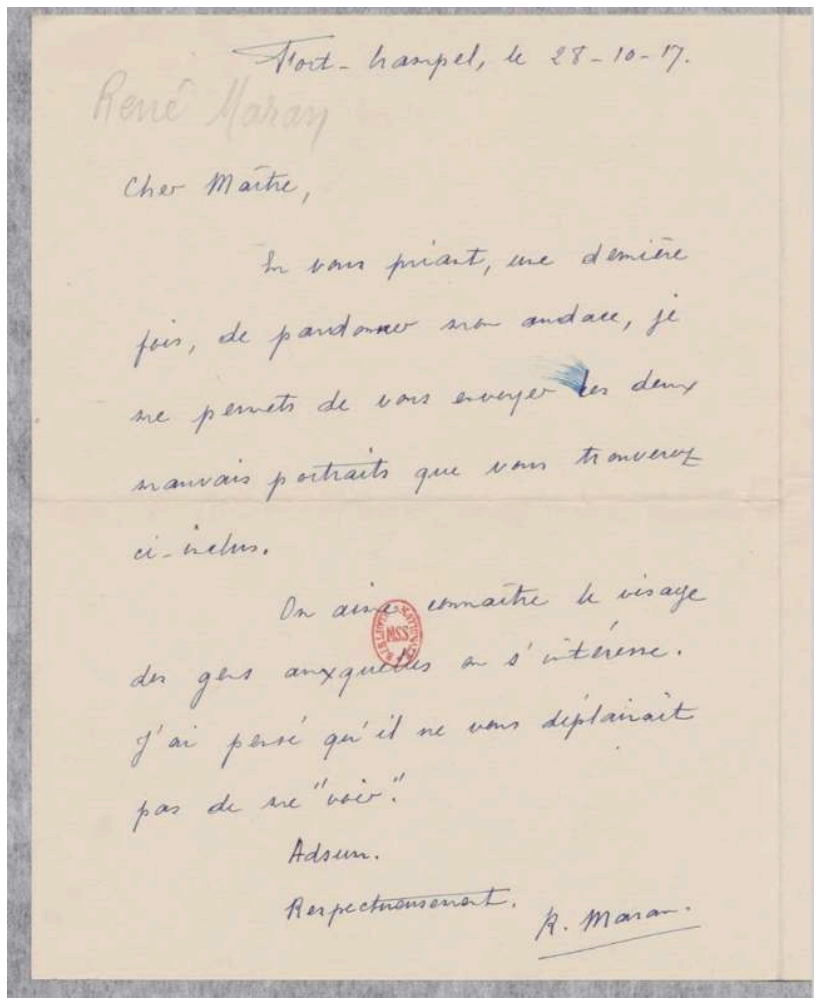

BnF, fonds Maurice Barrès (NAF 28210)

Fig. 6 : Portrait 1 de René Maran, envoyé à Maurice Barrès
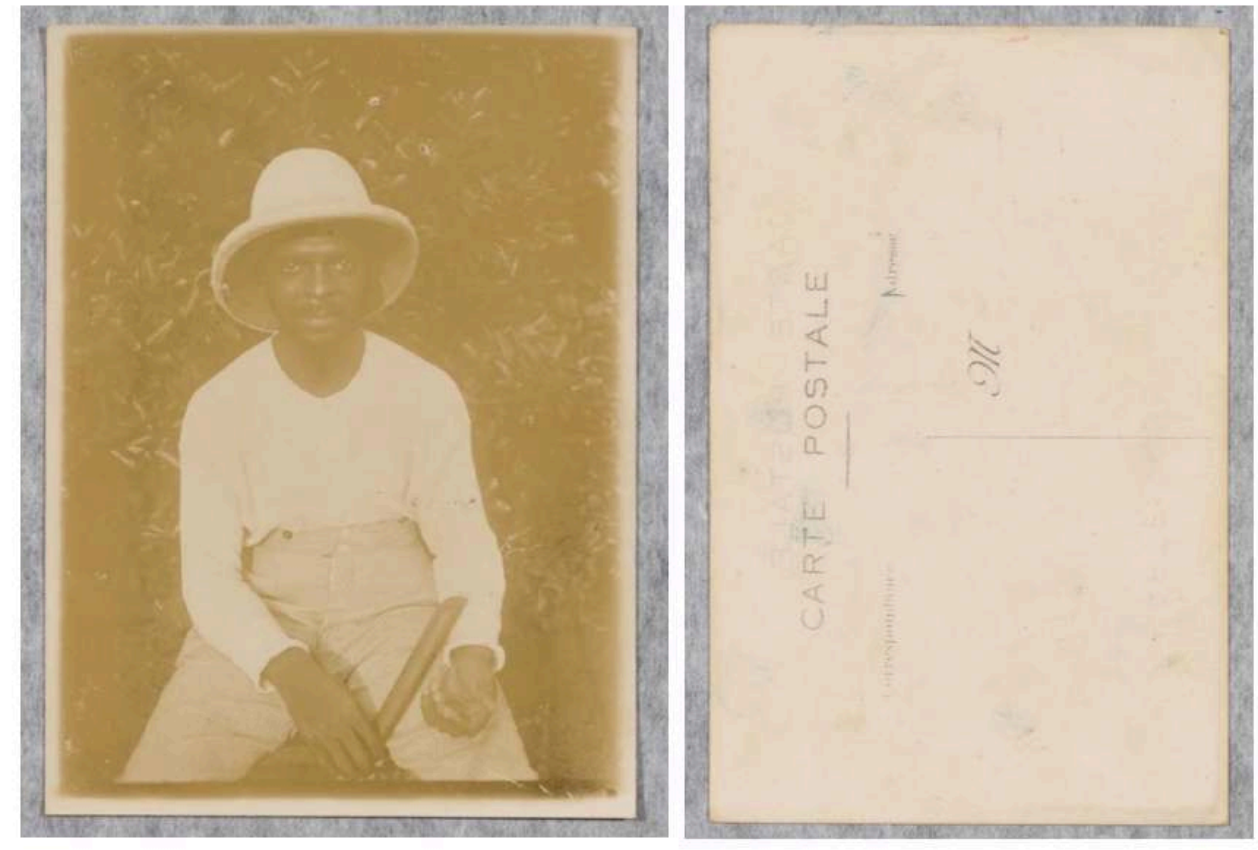

BnF, fonds Maurice Barrès (NAF 28210) 
Fig. 7 : Portrait 1 de René Maran, envoyé à Maurice Barrès

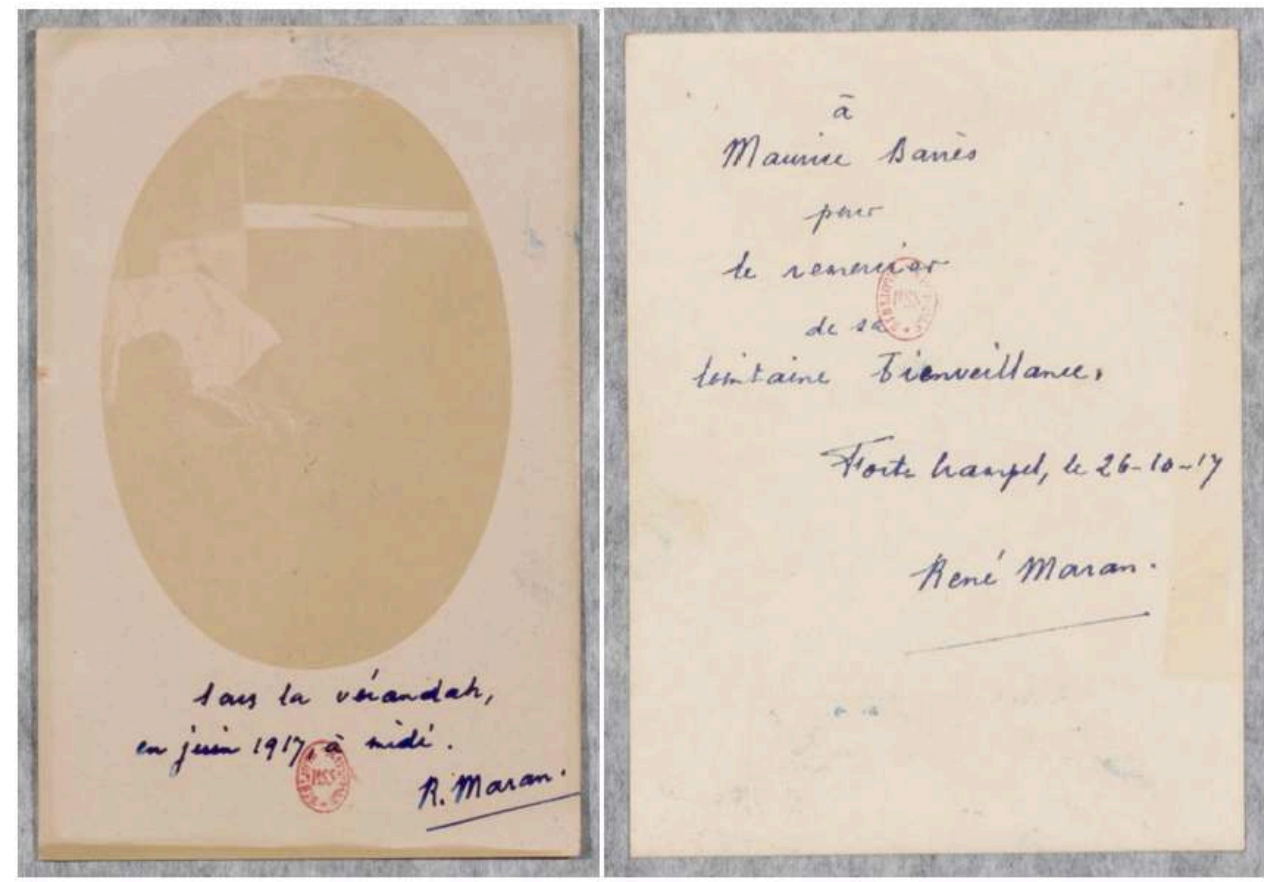

BnF, fonds Maurice Barrès (NAF 28210)

Ces lettres adressées à Barrès permettent de vérifier à quel point «le grand souci de Maran dès ses premiers jours au lycée de Talence, a été de prouver quelque chose, de se montrer l'égal de l'homme blanc, enfin d'arriver à être aussi Français que les Français de la métropole ${ }^{88}$ " comme le formule Femi Ojo-Ade. N'exprime-t-il pas son souhait de « [prouver] à ceux qui feignent de ne pas l'admettre que les nègres sont des hommes comme tout le monde "? Ne se pose-t-il pas "en défenseur d'une race qu'on a trop souvent avilie et que l'on abaisse sans motif »? Cette race, ajoute-t-il, "a cependant sacrifié bien de ses fils à la France et à la cause qu'elle défendait: la Civilisation ${ }^{89}$ ». Dans les papiers de l'écrivain conservés à la bibliothèque de l'université Cheikh Anta Diop à Dakar, Mbaye Gueye a mis au jour un manuscrit de ce qui deviendra Un homme pareil aux autres; il s'intitule "Un nègre parle ». Tina Harpin et Laura Gauthier Biasi, dans leur article, ont mis en lumière l'importance de ce manuscrit. On peut interpréter ce titre comme l'exercice d'un droit à la parole en tant que nègre promu sur « l'échelle de l'intelligence ${ }^{90} "$, les barreaux de cette échelle civilisationnelle correspondant aux stades de l'évolution.

Femi Ojo-Ade fait observer qu'« il a ainsi intériorisé la soi-disant question noire », d'où la " [difficulté] de s'identifier pleinement et de façon positive aux Noirs de son continent ancestral ${ }^{91} »$. Son départ fin 1909 pour l'AEF marque une étape charnière à la fois dans sa vie et sa carrière littéraire. Il marche alors sur les traces de son père, lequel avait été " appelé " par Savorgnan de Brazza, précisera-t-il en 1952 au cours d'un entretien radiophonique avec des anciens du lycée Montaigne de Bordeaux ${ }^{92}$. Ce voyage vers la «terre de [ses] ancêtres » mais, ajoute-t-il, « ancêtres [qu'il] réprouve parce [qu'il n'a] pas leur mentalité primitive ni leur goût ${ }^{93} "$, le bouleverse. Sa correspondance avec Manoel Gahisto, Léon Bocquet, notamment, en offre un vibrant témoignage. 

enjeux de la création artistique lorsqu'il explique « qu'elle fait contrepoids au complexe d'infériorité dont c'est la mission de toute colonisation que de l'instiller au colonisé101 ». Ne voir dans la posture de Maran qu'une symptomatologie de l'aliénation conduit à saper son auctorialité, donc à lui dénier toute autorité sur son œuvre, comme s’il n'avait été qu'un « névrosé, accidentellement noir » dont la couleur de peau ne serait qu'une «tentative d'explication d'une structure psychique ${ }^{102}$ »; en tant qu' "abandonnique noir", écrit Fanon dans le contexte de la Libération et des jugements pour faits de collaboration avec l'ennemi, René Maran alias Jean Veneuse serait «l'homme à abattre ${ }^{103}$ ». Or, dans les années 1920, s'il est bien un homme à 
abattre, c'est du point de vue des coloniaux, soient «tous ceux qui vivent des colonies " pour en tirer à peu près tout: "vivres frais et vivres secs, bois, plantes, que sais-je encore $^{104}$ ? » C'est ce qu'Olivier Sagna souligne à raison :

Comble du comble, il a osé remettre en cause la supériorité de l'homme blanc en déclarant tout à trac : « si l'inintelligence caractérisait le nègre, il n'y aurait que fort peu d'Européens ». Ces dénonciations et cette phrase assassine valurent à René Maran une solide inimitié de la part de ses ex-collègues ${ }^{105}$.

Mais dans le contexte de la décolonisation et des indépendances, le discours maranien devient obsolète. René Maran représente désormais l'archétype du nègre assimilé, cette « vieille négritude » dont Cahier d'un retour au pays natal entonne la disparition telle que l'avait annoncée Césaire dans L'Étudiant noir : « la jeunesse noire tourne le dos à la tribu des Vieux. / La tribu des Vieux dit : “assimilation", nous répondons : résurrection ${ }^{106}$ ! " Le «brillant illustrateur de l'humanisme noir ${ }^{107}$ » ainsi que le célébrait Senghor dans ce même numéro de L'Étudiant noir, devient, à rebours de l'Histoire, le " précurseur ${ }^{108}$ " d'une négritude dépassée par un mouvement panafricaniste symbolisé par la Guinée de Sékou Touré et le Ghana de Kwame Nkrumah ${ }^{109}$. Alors que l'engagement maranien pour la cause de ces " pauvres hères qui subissent notre civilisation ${ }^{110}$ » le faisait passer pour " anti-français ", du point de vue des coloniaux et de leurs partisans, celui-ci devient le symptôme d'une pathologie de l'aliénation, son œuvre étant désormais réduite à un document clinique ou ethnographique.

Alors que la Francophonie se met en place sous l'égide d'une coopération technique et économique avec, pour figure de proue, le président Senghor, la notion de négritude se mue en slogan politique et renvoie à une idéologie d'État - la Négritude comme humanisme et socialisme africain. Pernicieusement - ironie de l'histoire (littéraire) !-, celui qui se présentait comme " écrivain français » lié au terroir bordelais, contributeur à une revue régionaliste, Burdigala (1912-1914), lecteur de Maurice Barrès et admirateur de son engagement politique à la tribune de l'Assemblée nationale, a été naturalisé écrivain francophone. Son œuvre, relue en fonction de lieux communs postcoloniaux comme une "dénonciation du colonialisme ${ }^{111}$ ", est l'objet d'un profond malentendu préjudiciable à la compréhension de «l'affaire Batouala» et, plus largement, de l'histoire de la littérature française (et « francophone »).

Maran visait en effet « l'hypocrisie foncière de la France coloniale ", qu'il entendait, par le truchement de la LUDRN, « démasquer et rendre inoffensive afin que les populations d'outre-mer apprennent à aimer l'autre, la vraie [France], et se croient en tous lieux ses fils, et le proclament du fond de leur cœur, - malgré leur peau ${ }^{112}$ ». Il dénonçait l'attitude "impérialo-esclavagiste ou anti-libéral[e]», en cela "anti-française » du ministère des Colonies car devant mener inéluctablement à l'effondrement de l'empire, ce que lui, partisan de « la plus grande France ${ }^{113}$ ", s'ingéniait à prévenir.

\section{Conclusion}

La correspondance initiée par René Maran avec son aîné Maurice Barrès invite à une lecture croisée avec ses fictions romanesques. De cette lecture transversale peuvent se déduire une intention et une mise en scène auctoriale : l'intention de révéler des faits à l'opinion publique et la mise en scène d'un critérium de vérité soutenant cette révélation. 
Sans doute y aurait-il donc lieu de questionner la mise en garde du narrateur Jean Veneuse - à quel point est-il fiable ? - lorsqu'il nous prévient que son récit « constitue moins un livre à thèse ou un état de race qu'un état d'âme "; d'autant que, plus loin, il nous le présente comme le "voyage d'une race à une autre et d'un cœur à un autre cœur» et souhaite, par ce moyen littéraire, «imposer silence aux préjugés, aux sophismes et aux partis pris, qui cherchent à transformer les arrêts changeants de sciences trop souvent faillibles en autant d'impératifs ethniques ${ }^{114} »$. De fait, ce dernier apparaît comme le porte-parole de l'auteur Maran lorsqu'il fait dire à son héros Veneuse «ne [savoir] bien qu'une chose : c'est que le nègre est un homme comme les autres" - "des hommes comme tout le monde " écrit-il à Barrès en soulignant l'expression - phrase citée dans Peau noire, masques blancs. Aussi Mbougar Sarr, préfacier de la réédition du roman à l'occasion du centenaire du prix Goncourt 1921, a$\mathrm{t}$-il sans doute raison d'entendre dans le titre « un écrivain pareil aux autres ${ }^{115}$ ». Cela, dans la mesure où « il semble marqué par cette intention de le rabaisser, qui contredit la volonté exprimée officiellement d'élever l'homme de couleur" comme l'analyse André Fraisse. Pour ce dernier, "il réagit en devenant écrivain ${ }^{116}$ ». Mbougar Sarr abonde dans ce sens : «Emprisonné comme homme dans les rets du discours racialiste, il a tenté de s'en sortir comme écrivain, dans la solitude de la création et l'indépendance des vues ${ }^{117}$. » Toutefois, il semble que le sens de l'écriture de Maran, s'il n'est pas réductible à une idéologie, tienne cependant à une politique - suscitée par cette " cause " au sujet de laquelle il interpelle son homologue Barrès - dont la littérature serait le lieu d'expression privilégié. Si donc Un homme pareil aux autres doit se lire comme une fiction, c'est bel et bien celle d'un autre que soi, un double littéraire qui met en abyme les conditions de sa propre réception. Dans la préface de Batouala, Maran prévient et nous annonce :

Mon livre n'est pas de polémique. Il vient, par hasard, à son heure [je souligne]. La question nègre est « actuelle $»^{118}$.

Et les trois premières phrases d'Un homme pareil aux autres font écho au discours préfaciel de Batouala.

Je m'appelle Jean Veneuse. Le nègre que je suis a peut-être tort de publier les confidences qu'on va lire. On m'a pourtant conseillé de le faire, parce que le moment semble venu [je souligne] d'en saisir l'opinion publique ${ }^{119}$.

La fiction maranienne se place résolument sous le signe d'une actualité socio-politique. En ce sens, le «Je m'appelle » devient l'acte performatif d'un dédoublement littéraire, geste inaugural de l'œuvre romanesque de Maran. Dans l'incipit du Cœur serré le héros se présente à nous :

Je m'appelle Georges Lindre. Mon nom, qui sonne clair, est un nom français.

Qu'on me pardonne ce cri d'orgueil, dont je m'excuse. Français, je le suis, mais ce n'est pas en France que j'ai vu le jour ${ }^{120}$.

Ce sont les mots et les idées de Maran, militant pour l'abolition du régime esclavagiste et ségrégationniste consacré par le code de l'indigénat et les circulaires ministérielles, que portent Georges Lindre et Jean Veneuse. C'est ainsi qu'il faut comprendre la dissociation établie entre l'auteur et ses personnages: si, dans la préface de Batouala, l'auteur Maran soutient être allé jusqu'à s'absenter de son texte ${ }^{121}$, il annonce des prises de parole en son nom propre : «je parlerai en mon nom et non pas au nom d'un autre; ce seront mes idées que j'exposerai et non pas celles d'un autre ${ }^{122}$.» Cet autre-là c'est Batouala, puis Lindre, Veneuse, le héros d'Un homme pareil aux autres qui déplore la 
perversion de l'œuvre coloniale française entre les mains de «professionnels du mensonge, ceux dont la duplicité est le métier ${ }^{123}$ ». Et pourtant...

Fonctionnaire colonial... Ce métier aurait pu être si beau, si généreux, si noble! Hélas ! La colonisation est une déesse âpre et cruelle, qui ne se paie pas de mots et se nourrit de sang. Trop pratique pour être sensible, rien ne la détourne de ses projets. Elle se fonde sur l'injustice et l'arbitraire. [...] Il faut, pour lui plaire, arrêter des innocents. Ni les uns ni les autres n'ont quoi que ce soit à se reprocher. Mais la main-d'œuvre pénale faisant plus ou moins défaut, cette illégalité permet d'entreprendre ou d'achever, sans grever le moins du monde le budget local, les travaux en cours. La force primant le droit, le meurtre célébré et honoré, c'est ça, la colonisation. Il n'est personne qui ne le sache. Alors, pourquoi se plaît-on à jouer sur les mots? Pourquoi n'a-t-on pas le droit de dire à tous, la vérité que l'on détient? Et pourquoi, lorsqu'on ne fait que relater une faible partie de ce qui est patent, soulève-t-on tant de dénégations inutiles et tant de haines ${ }^{124}$ ?

En ce paragraphe se trouvent résumées l'amertume et la désillusion d'un jeune Maran, pétri d'idéalisme - des chimères?

Dans une lettre à Mercer Cook de 1948, il s'exclame à propos de l'écrivain Raphaël Tardon, ce "jeune martiniquais extrêmement doué » dont le "teint est d'un bistre assez clair » et qui a, de toute évidence, du «sang noir dans les veines » qu'il est «le premier à le proclamer»: "Que de chemin parcouru, depuis que j'apprends aux Français de couleur à ne pas avoir honte de leur race !125 ». Par ces mots confiés à son ami d'outre-Atlantique, Maran se félicite des fruits de son engagement à défendre " une race qu'on a trop souvent avilie » en se posant comme le pionnier d'une négritude que son œuvre aura largement contribué à mettre au-devant de la scène. Ainsi peut-il faire dire au héros de son Journal sans date, dans une édition augmentée et titrée Un homme pareil aux autres, que « le moment semble venu d'en saisir l'opinion publique ${ }^{126} »$. De sa première lettre à Barrès à cette édition finale ayant connu plusieurs titres, trente ans se sont écoulés; le processus devant conduire aux déclarations d'indépendance des colonies européenne est entériné par la signature, en juin 1945, de la Charte des Nations Unies qui oblige, dans son article 73, les États signataires à enclencher ou favoriser un processus de décolonisation en reconnaissant la " primauté des habitants » des territoires colonisés. Dès lors, la relecture de l'œuvre de René Maran au prisme de la négritude senghorienne et d'une symptomatologie de l'aliénation apparaît significative de ces «nombreux malentendus » et de ces « acrobaties périlleuses qui ont transformé le "grand cri nègre" en une clameur "pervertie" 127 ». Or, l'écrivain fut l'un de ces géants sur les épaules desquels nous sommes assis, lui qui proclamait être « de ceux qui croient au rôle social de l'écrivain, contre ces faiseurs et ces baladins qui passent, aveugles et sourds, devant les problèmes lourds d'inconnues qui émeuvent actuellement le monde civilisé128. » Plutôt que parler d'aliénation, qui consiste à enfermer l'auteur dans une clinique du sujet et à ériger le lecteur en surplomb de l'œuvre, la notion d'assimilation, entendue comme dialectique, invite à porter l'attention sur les stratégies discursives mises en œuvre par l'écrivain. De fait, c'est bel et bien dans cette perspective que René Maran initie une correspondance avec Maurice Barrès dont il sollicite l'« appui » pour défendre une "cause " et démontrer une « thèse » : en tant qu' « écrivain français ». 


\section{BIBLIOGRAPHIE}

ANSOM 8 APOM 1 - Archives privées d'outremer d'André Fraisse.

« Potaches et labadens ou la classe intemporelle - Le Lycée Montaigne à Bordeaux ( $1^{\text {re }}$ diffusion : 10/02/1952 Chaîne Nationale) ", Radio France Culture, 11 août 2018, https://

www.franceculture.fr/emissions/les-nuits-de-france-culture/potaches-et-labadens-ou-la-classeintemporelle-le-lycee-montaigne-a-bordeaux-1ere-diffusion-10021952.

« Vote des femmes », 1935, Bibliothèque Marguerite Duras, DOS 396 VOT, https://bibliothequesspecialisees.paris.fr/ark:/73873/pf0001952392/0024?posInSet=30\&queryId=0b2bfc49-a705-4e6fb6ad-3d345e78c46c.

Anonyme, «Le nègre René Maran et la question noire », La Vague, nº 208, 22 décembre 1921, p. 1-2.

BALANDIER Georges, « Les mythes politiques de colonisation et de décolonisation en Afrique », Cahiers Internationaux de Sociologie, XXXXIII, I, 1963, p. 87-94.

BARRÈs Maurice, Le Roman de l'énergie nationale, Paris, E. Fasquelle, coll. « BibliothèqueCharpentier ", 1897, 3 vol.

BOCQUET Léon, « Préface », dans René Maran, Le Petit roi de Chimérie, Paris, A. Michel, 1924, p. 7-63.

CÉSAIRE Aimé, « Négreries : jeunesse noire et assimilation », dans Albert James Arnold (éd.), Poésie, théâtre, essais et discours, Paris, Agence universitaire de la francophonie, CNRS éditions et Présence africaine, coll. « Planète libre », $\mathrm{n}^{\circ}$ 4, 2013, 1 vol., p. 1292-1294.

CÉSAIRE Aimé, « L'homme de culture et ses responsabilités », dans Albert James Arnold (éd.), Poésie, théâtre, essais et discours, Paris, Agence universitaire de la francophonie, CNRS éditions et Présence africaine, coll. « Planète libre », $\mathrm{n}^{\circ}$ 4, 2013, 1 vol., p. 1553-1559.

CÉSAIRE Aimé, Écrits politiques, Paris, J.-M. Place, 2013.

CÉSAIRE Aimé, Cahier d’un retour au pays natal, Paris, Présence africaine, 1956.

FABRE Michel, « Autour de Maran », Présence Africaine, n 86, 1973, p. 165-172.

FANON Frantz, Peau noire, masques blancs, Paris, Éditions du Seuil, 1952.

FONKOUA Romuald-Blaise, Aimé Césaire, 1913-2008, Paris, Perrin, 2010.

FRAISSE André, « René Maran », dans Hommage à René Maran, Paris, Présence africaine, 1965, p. 271-276.

GAHISTO Manoel, «La genèse de Batouala », dans Hommage à René Maran, Paris, Présence africaine, 1965, p. 93-155.

KISUDIKI Nadia Yala, « Négritude et philosophie », Rue Descartes, n 83, 2014, p. 1-10.

LAURU Didier, « Dépersonnalisation, le doute d'exister ? ", Figures de la psychanalyse, vol. 9, n 1 , Érès, 2004, p. 87-95.

LONDRES Albert, Terre d'ébène (la Traite des noirs), Paris, Albin Michel, 1929.

MALELA Buata B., Les Écrivains afro-antillais à Paris, 1920-1960 : stratégies et postures identitaires, Paris, Éd. Karthala, coll. « Lettres du Sud », 2008. 
MARAN René, « Lettre inédite de René Maran à André Fraisse », Francofonía, n 14 , Servicio de Publicaciones, rubrique « Francofonía », 2005, p. 11-13.

MARAN René, « Journal », dans W. Mercer Cook (éd.), Hommage à René Maran, Paris, Présence africaine, 1965, p. 289-305.

MARAN René, Un homme pareil aux autres, Paris, Éditions Arc-en-ciel, 1947.

MARAN René, « Un petit-neveu de Chatterton : Jean-Joseph Rabearivelo », Le Monde illustré, 21 août 1937, p. 579.

MARAN René, « Racisme et civilisation », Le Nouvelliste d'Indochine, 12 septembre 1936, p. 4.

MARAN René, Le Cour serré, Paris, A. Michel, 1931.

MARAN René, Le Tchad: de sable et d'or, Paris, Librairie de la Revue française ; Alexis Redier, coll. «Toutes nos colonies ", $\mathrm{n}^{\circ} 4,1931$.

MARAN René, lettre à Marcel Martinet, Bibliothèque nationale de France, fonds Marcel Martinet, NAF 28352 / f. 214.

MARAN René, « Djogoni », Candide, nº 199, 5 janvier 1928, p. 5, 8.

MARAN René, «Journal sans date », dans Les Euvres libres, Arthème Fayard et Cie, sans lieu, 1927, p. 105-236.

MARAN René, « Ma condamnation », Les Continents, 15 décembre 1924.

MARAN René, « Au pied du mur », Les Continents, 15 juillet 1924, p. 1.

MARAN René, Le Petit roi de Chimérie, Paris, A. Michel, 1924.

MARAN René, Batouala : véritable roman nègre, Paris, A. Michel, 1921.

MARAN René, «Préface », dans Batouala : véritable roman nègre, Paris, A. Michel, 1921, p. 9-18.

MARAN René, lettres à Maurice Barrès, Bibliothèque nationale de France, fonds Maurice Barrès, NAF 28210.

MARAN René, lettres à Manoel Gahisto, archives privées de Bernard Michel.

MBOUGAR SARR Mohamed, « Préface », dans René Maran, Un homme pareil aux autres, Marseille, Typhon, 2021, p. 11-22.

OJO-ADE Femi (éd.), René Maran : écrivain négro-africain, Paris, F. Nathan, coll. « Classiques du monde », 1977.

RENAULT Mathieu, « Amour de la race ou amour au-delà des races ? Frantz Fanon, lecteur de René Maran ", Présence Africaine, ${ }^{\circ}$ 187-188, 9 août 2013, p. 231-244.

SAGNA Olivier, Des pionniers méconnus de l'indépendance : Africains, Antillais et luttes anti-colonialistes dans la France de l'entre-deux-guerres (1919-1939), Université Paris VII Diderot, 1986.

SAPIRO Gisèle, Peut-on dissocier l'œuvre de l'auteur ?, Paris, France, Seuil, 2020.

SENGHOR Léopold Sédar, « Avant-propos », dans Hommage à René Maran, Paris, Présence africaine, 1965.

SENGHOR Léopold Sédar, « Avant-propos », dans Hommage à René Maran, Paris, Présence africaine, 1965.

SENGHOR Léopold Sédar, « L'humanisme et nous : “René Maran” ", L'Étudiant noir, 1935. 
SYLVAIN Benito, Du Sort des indigènes dans les colonies d'exploitation, Paris, L. Boyer, 1901.

TAGORE Rabindranath, « Le Message de l'Inde au Japon », Andrée Jouve (trad.), dans Romain Rolland, A la civilisation, sans lieu, 1917, p. 25-30.

TOUMSON Roger, La Transgression des couleurs : littérature et langage des Antilles, XVIII ${ }^{e}, \mathrm{XIX}^{e}, \mathrm{XX}^{e}$ siècles, Paris, Éd. caribéennes, 1989, 2 vol

VIOLAINES René, « Mon ami René Maran : sa vie et son œuvre à travers ses lettres et mes souvenirs », dans Hommage à René Maran, Paris, Présence africaine, 1965, p. 15-41.

\section{ANNEXES}

\section{Première lettre de René Maran à Maurice Barrès}

René Maran

adjoint de $2^{\mathrm{e}}$ classe des Services Civils

à

Fort-Sibut

Oubangui-Chari

Afrique Équatoriale Française

Monsieur et cher Maître

Cette lettre d'une écriture inconnue, venant de si loin, vous étonnera sans doute. Et vous serez plus étonné encore lorsque, l'ayant lue, vous saurez qu'elle émane d'un nègre martiniquais, qui à vrai dire ne connaît que peu son pays d'origine puisque, atteignant la trentaine, il n'a passé à Fort-de-France que les trois premières années de sa vie.

Je ne vous écris que pour plaider une cause - parce que je suis nègre. En effet, pour moi, l'une des tristesses de cette guerre est que de plus en plus l'on considère mes frères en couleur comme des mercenaires, non comme des êtres perfectibles. Et pourtant... Bocquet, Despaix, Lafon, Jean Balde, Lebesgue, tous mes amis littéraires, depuis qu'ils me connaissent, ne pensent plus ainsi. Ils voient ce que l'instruction peut faire d'un primitif. Et, de ce qu'ils me considèrent, ou me considéraient comme un des leurs, - car, hélas ! plusieurs d'entre eux sont morts, - je suis extrêmement fier. Il me semble, mauvais orgueil, - que je réhabilite une race trop avilie, trop humiliée. Ai-je tort? Si donc je vous écris, ce n'est pas que je veuille que vous fassiez le panégyrique de ces pauvres nègres, mais bien plutôt pour que vous mettiez votre génie et votre mesure à leur service. Ils méritent mieux qu'on ne leur donne. Ah ! si vous vouliez le dire bien haut, tout le monde vous croirait.

Je vous envoie quelques poèmes qui m'ont été inspirés par la guerre. Je ne les crois pas plus mauvais que ceux que j'ai pu lire jusqu'ici. Je ne vous les envoie que pour vous confirmer la thèse que je soutiens, et qui est que les nègres ont une intelligence qui ne demande qu'à se développer.

S'il était possible, je voudrais que ces stances, sous votre égide, pussent paraître à une des grandes revues littéraires qui honorent Paris et le monde. Mais sachez bien que je n'œuvre que pour ma propre satisfaction, et qu'il me serait indifférent, si elles venaient à paraître, que ces stances, qui ont pour le moins le mérite d'une claire sincérité, ne fussent pas signées de mon nom. Toutefois, faites au gré de votre jugement. Si elles vous agréaient, en récompense, ne faites pas état de ma lettre en quoi que ce soit. Je ne 
suis qu'un élève qui écrit à un de ses Maîtres en le priant de consacrer son crédit à défendre une cause juste entre toutes. Considérez cette lettre à ce seul point de vue. Et je serai amplement payé de toute ma peine si j'ai pu vous émouvoir, vous convaincre... En vous priant de bien vouloir excuser ma lointaine audace, veuillez agréer, Monsieur et cher Maître, mes saluts les plus respectueux.

R. Maran.

Le 12 janvier 1917

\section{NOTES}

1. L. BOCQUET, « préface ", René Maran, Le Petit roi de Chimérie, Paris, Albin Michel, 1924, p. 12.

2. Je tiens à remercier le conservateur Jérome Villeminoz pour avoir permis la consultation des lettres et des tapuscrits joints.

3. M. BARRÈs, Le Roman de l'énergie nationale, Paris, E. Fasquelle, 1897, 3 vol.

4. L. S. SENGHOR, « L'humanisme et nous: "René Maran" ", L'Étudiant noir, 1935.

5. R. VIOLAINES, "Mon ami René Maran: sa vie et son œuvre à travers ses lettres et mes souvenirs ", dans Hommage à René Maran, Paris, Présence Africaine, 1965, p. 20.

6. Ibid.

7. R. MARAN, lettre à M. Barrès, 12 janvier 1917, f. 1 .

8. G. LECLERC, Anthropologie et Colonialisme: essai sur l'histoire de l'africanisme, Paris, Fayard, 1972, p. 28.

9. M. FABRE, « Autour de Maran », Présence africaine, $n^{\circ}$ 86, 1973, p. 165.

10. L. S. SENGHOR, « Avant-propos ", dans Hommage à René Maran, Paris, Présence Africaine, 1965.

11. N. Y. KISUDIKI, « Négritude et philosophie ", Rue Descartes, n 83, 2014, p. 1.

12. A. CÉSAIRE, « Négreries : jeunesse noire et assimilation », dans A. J. Arnold (éd.), Poésie, théâtre, essais et discours, Paris, Agence universitaire de la francophonie, CNRS éditions et Présence Africaine, 2013, 1 vol., p. 1292-1294.

13. A. CÉSAIRE, Cahier d'un retour au pays natal, Paris, Présence Africaine, 1956

14. N. Y. KISUDIKI, « Négritude et philosophie », op. cit., p. 4

15. Id.

16. "La théorie de la négritude contribue à imposer l'exigence, maintenant très généralisée, de "reconstruction de la personnalité africaine". Lorsque les chefs d'État reconnaissent comme une de leurs tâches cette obligation de reconstruire la personnalité africaine, ils rendent d'une certaine manière hommage à la théorie de la négritude. C'est le cas pour M. Sékou Touré.» G. BALANDIER, "Les mythes politiques de colonisation et de décolonisation en Afrique », Cahiers Internationaux de Sociologie, XXXXIII, I, 1963, p. 87-94.

17. R. Toumson, La Transgression des couleurs : littérature et langage des Antilles, XVIII ${ }^{e}, X I X^{e}, X X^{e}$ siècles, Paris, Éd. caribéennes, 1989, p. 371-372, t. 2.

18. R.-B. FonkouA, Aimé Césaire, 1913-2008, Paris, Perrin, 2010, p. 52.

19. M. RENAULT, «Amour de la race ou amour au-delà des races? Frantz Fanon, lecteur de René Maran », Présence africaine, $\mathrm{n}^{\circ}$ 187-188, 2013, p. 232.

20. Dans les collections de la bibliothèque Marguerite Duras où sont conservés les numéros de La Vague, hebdomadaire fondé en 1918 par Pierre Brizon et Marcelle Capy qui se présente comme " socialiste, pacifiste et féministe ", existe un dossier documentaire intitulé « Vote des femmes ", DOS 396 VOT, https://bibliotheques-specialisees.paris.fr/ark:/73873/pf0001952392/0024? posInSet=30\&queryId=0b2bfc49-a705-4e6f-b6ad-3d345e78c46c. Dans celui-ci se trouve, page 178, une coupure presse qui semble dater de 1935 dans laquelle est reproduite une réponse de René Maran, initialement publiée dans L'Essor féminin, en faveur du droit de vote des femmes. C'est 
dans ce cadre que l'écrivain met en parallèle l'octroi du droit de vote aux esclaves transformés en citoyens français par le décret d'abolition de l'esclavage de 1848 et le refus du droit de vote aux femmes qui sont pourtant citoyennes françaises et, qui plus est, de plus longue date fait valoir René Maran : «Admettez-vous que, dans le foyer qui l'a recueilli, le fils adoptif ait des droits supérieurs à ceux des filles légitimes ? " Pour lui, les femmes - blanches faut-il entendre - seraient plus légitimes à jouir du droit de vote que les hommes d'ascendance africaine; et de s'en indigner : "Que diriez-vous d'un père et d'une mère qui légueraient la plus grosse part de leur héritage à l'enfant recueilli et qui trusteraient ainsi leur fille légitime. De toute façon, le fisc réparerait l'injustice, mais hélas! à son propre profit. / Et voici cependant ce qui se passe en France, les fils adoptifs, les fils d'une autre race et les fils naturalisés, parce qu'ils ont la chance d'être des hommes, jouissent des privilèges que l'on refuse aux filles qui sont cependant des enfants du terroir. "

21. A. CÉSAIRE, « Négreries : jeunesse noire et assimilation », art. cit.

22. Expression employée lors du séminaire René Maran 2020-2021, Cf. B. B. MALELA, Les Écrivains afro-antillais à Paris, 1920-1960 : stratégies et postures identitaires, Paris, Éd. Karthala, 2008.

23. A. CÉSAIRE, « Négreries : jeunesse noire et assimilation », art. cit.

24. A. CÉSAIRE, «L'homme de culture et ses responsabilités ", dans A. J. Arnold (éd.), Poésie, théâtre, essais et discours, Paris, Agence universitaire de la francophonie, CNRS éditions et Présence Africaine, 2013, 1 vol., p. 1554-1555.

25. G. SAPIRO, Peut-on dissocier l'oeuvre de l'auteur?, Paris, France, Seuil, 2020, p. 31.

26. F. FANON, Peau noire, masques blancs, Paris, Seuil, 1952, p. 43.

27. ANSOM 8 APOM 1 - Archives privées d'outre-mer d'André Fraisse.

28. Id.

29. D'après la transcription d'une lettre en date du 10 juin 1946 adressée à Mercer Cook communiquée par Roger Little au sein de l'équipe René Maran de l'ITEM.

30. A. CÉSAIRE, Écrits politiques, Paris, J.-M. Place, 2013, p. 65.

31. O. SAGNA, Des pionniers méconnus de l'indépendance: Africains, Antillais et luttes anti-colonialistes dans la France de l'entre-deux-guerres (1919-1939), Université Paris VII Diderot, 1986, p. 235-236.

32. R. MARAN, lettre à Maurice Barrès, 13 août 1917, f. 3.

33. R. TAGORE, «Le Message de l'Inde au Japon », A. Jouve (trad.), dans R. Rolland, A la civilisation, s. 1., 1917, p. 25-30

34. R. MARAN, « Racisme et civilisation », Le Nouvelliste d'Indochine, 12 septembre 1936, p. 4.

35. R. MARAN, «Préface », dans Batouala : véritable roman nègre, Paris, A. Michel, 1921, p. 12.

36. R. MARAN, lettre à Maurice Barrès, 28 février 1918, f. 1-2.

37. Ibid., f. 4.

38. Ibid., f. 5 .

39. R. MARAN, « Préface ", déjà citée, p. 8.

40. Dans le chapô de l'article, sont évoquées les menaces de mort ayant plané sur l'écrivain et les accusations calomnieuses ayant été proférées à son encontre afin de le faire taire : « Au cours du procès des Continents, M. Blaise Diagne reproche à notre ami d'avoir été condamné, en 1919, par le Tribunal correctionnel de Bangui, à une peine de 50 francs d'amende, avec bénéfice de la loi de sursis. / Cette condamnation gêne si peu René Maran, que c'est dans le dessein de faire sur elle la pleine lumière qu'il a écrit en novembre 1918, sa préface de Batouala.» R. MARAN, "Ma condamnation », Les Continents, 15 décembre 1924.

41. R. MARAN, «Lettre inédite de René Maran à André Fraisse ", Francofonía, $n^{\circ}$ 14, 2005, p. 11-13.

42. Anon., «Le nègre René Maran et la question noire ", La Vague, $n^{\circ}$ 208, 22 décembre 1921, p. 1-2.

43. R. MARAN, lettre à M. Barrès, 28 février 1918.

44. R. MARAN, « Djogoni », Candide, $\mathrm{n}^{\circ} 199,5$ janvier 1928, p. 5, 8 . 
45. M. GAHISTO, «La genèse de Batouala », dans Hommage à René Maran, Paris, Présence Africaine, 1965, p. 129.

46. D'après un corpus d'extraits de lettres adressés à Manoel Gahisto concernant ce récit, un corpus établi par Roseline Garcia Ballester dans le cadre du séminaire René Maran de l'ITEM. R. MARAN, lettre à Manoel Gahisto, 7 mars 1914.

47. R. MARAN, lettre à Maurice Barrès, 12 janvier 1917, f. 1-2.

48. Ibid., f. 3.

49. Ibid., f. 3-4 (voir fig. 3).

50. R. MARAN, lettre à M. Barrès, 28 février 1918, p. 1

51. «[...] l'on sait ce qui se passe en ces terres lointaines et [...], jusqu'ici, l'on n'a pas essayé de remédier aux abus, aux malversations et aux atrocités qui y abondent. », R. MARAN, " Préface », op. cit., p. 12

52. R. MARAN, Un homme pareil aux autres, op. cit., p. 11

53. R. MARAN, « Préface », déjà citée, p. 13

54. R. MARAN, lettre à M. Barrès, 8 décembre 1918, p. 2

55. R. MARAN, lettre à M. Barrès, 12 janvier 1917, p. 1

56. R. MARAN, lettre à M. Barrès, 19 juillet 1917, p. 3

57. R. MARAN, lettre à M. Barrès, 8 décembre 1918, p. 2

58. R. MARAN, Un homme pareil aux autres, op. cit., p. 16

59. Voir leur article dans ce même numéro, https://journals.openedition.org/coma/7444.

60. R. MARAN, lettre à M. Barrès, 28 février 1918, p. 3

61. D. LAURU, «Dépersonnalisation, le doute d'exister?», Figures de la psychanalyse, vol. 9, nº 1 , Érès, 2004, p. 87-95.

62. R. MARAN, « Préface ", déjà citée, p. 13.

63. Id.

64. R. MARAN, lettre à M. Barrès, 12 janvier 1917, op. cit.

65. Expression employée par Buata Malela au cours du séminaire René Maran de l'ITEM.

66. R. MARAN, Un homme pareil aux autres, op. cit., p. 182, 184

67. F. FANON, Peau noire, masques blancs, op. cit., p. 208.

68. R. MARAN, lettre à M. Barrès, 8 décembre 1918, p. 2

69. R. MARAN, « Préface ", déjà citée, p. 11

70. R. MARAN, lettre à M. Barrès, 28 février 1918, f. 6

71. R. MARAN, lettre à M. Barrès, 8 décembre 1918, f. 2 .

72. R. MARAN, lettre à M. Barrès, 28 février 1918, f. $2,4$.

73. R. MARAN, lettre à M. Barrès, 19 juillet 1917, f. 6.

74. R. MARAN, « Journal », dans W. M. Cook (éd.), Hommage à René Maran, Paris, Présence Africaine, 1965, p. 304.

75. R. MARAN, « Préface ", déjà citée, p. 15.

76. Tapuscrit annoté d'une causerie radiophonique de décembre 1937 conservé dans les archives familiales de l'écrivain.

77. R. VIOLAINES, «Mon ami René Maran: sa vie et son oeuvre à travers ses lettres et mes souvenirs », art. cit., p. 20.

78. R. MARAN, lettre à M. Barrès, 8 décembre 1918, f. 1 .

79. R. MARAN, lettre à M. Barrès, 28 mai 1920, f. 1-2.

80. R. MARAN, lettre à M. Barrès, 12 janvier 1917, f. 3.

81. R. MARAN, « Au pied du mur ", Les Continents, 15 juillet 1924, p. 1.

82. B. SYLVAIN, Du sort des indigènes dans les colonies d'exploitation, Paris, L. Boyer, 1901, p. 307.

83. A. LONDRES, Terre d'ébène (la Traite des noirs), Paris, Albin Michel, 1929, p. 51. 
84. R. MARAN, lettre à M. Barrès, 13 août 1917 f. 1 .

85. R. MARAN, lettre à M. Barrès, 8 décembre 1918.

86. R. MARAN, lettre à M. Barrès, 28 mai 1920, f. 1-2

87. R. MARAN, lettre à Maurice Barrès, 28 octobre 1917.

88. F. OJO-ADE (éd.), René Maran : écrivain négro-africain, Paris, F. Nathan, 1977, p. 15

89. R. MARAN, lettre à M. Barrès, 28 mai 1920, f. 1-2.

90. R. MARAN, lettre à M. Barrès, 8 décembre 1918, f. 2.

91. F. oJO-ADE, op. cit., p. 16.

92. «Potaches et labadens ou la classe intemporelle - Le Lycée Montaigne à Bordeaux (1ère diffusion : 10/02/1952 Chaîne Nationale)", Radio France Culture, 11 août 2018, https:// www.franceculture.fr/emissions/les-nuits-de-france-culture/potaches-et-labadens-ou-la-classeintemporelle-le-lycee-montaigne-a-bordeaux-1ere-diffusion-10021952.

93. L. BOCQUET, « Préface », dans R. Maran, Le Petit roi de Chimérie, op. cit., p. 15

94. R. MARAN, Le Tchad: de sable et d'or, Paris, Librairie de la Revue française ; Alexis Redier, 1931, p. 135

95. F. OJO-ADE (éd.), op. cit., p. 16

96. Ibid., p. 15

97. Pour lui, Maran est partagé «entre la sympathie instinctive qu'il ressent pour ses frères demeurés barbares et les obligations secrètes qu'il a envers la civilisation qui lui a donné une âme et un cerveau de blanc singulièrement cultivé ». C'est ainsi qu'il conçoit le "conflit des races » dont Maran serait la proie. Il rapporte aussi qu'il pouvait avoir «l'air de s'excuser» de sa « race ». L. BOCQUET, « Préface », op. cit., p. 10, 15

98. R. MARAN, lettre à M. Barrès, 12 janvier 1917, f. 1-3

99. R. MARAN, Un homme pareil aux autres, op. cit., p. 18-19

100. R. MARAN, lettre à M. Barrès, 12 janvier 1917, f. 1-3.

101. A. CÉSAIRE, « L'homme de culture et ses responsabilités », op. cit., p. 1554.

102. F. FANON, Peau noire, masques blancs, op. cit., p. 86-87.

103. Ibid., p. 75.

104. R. MARAN, lettre à M. Barrès, 19 juillet 1917, f. 3-5.

105. O. SAGNA, Des pionniers méconnus de l'indépendance: Africains, Antillais et luttes anti-colonialistes dans la France de l'entre-deux-guerres (1919-1939), op. cit., p. 223.

106. A. CÉSAIRE, « Négreries : jeunesse noire et assimilation », art. cit., p. 1293.

107. L. S. SENGHOR, « L'humanisme et nous : "René Maran" », L'Étudiant noir, 1935.

108. L. S. SENGHOR, «Avant-propos ", dans Hommage à René Maran, Paris, Présence Africaine, 1965.

109. G. BALANDIER, «Les mythes politiques de colonisation et de décolonisation en Afrique », art. cit.

110. R. MARAN, « Au pied du mur », art. cit.

111. C'est le sous-titre du colloque d'hommage organisé par la Collectivité territoriale de Martinique le 15 janvier 2021.

112. R. MARAN, « Au pied du mur », art. cit.

113. R. MARAN, «Un petit-neveu de Chatterton: Jean-Joseph Rabearivelo", Le Monde illustré, 21 août 1937, p. 579.

114. R. MARAN, Un homme pareil aux autres, op. cit., p. 12-13.

115. M. MBOUGAR SARR, «Préface", dans R. Maran, Un homme pareil aux autres, Marseille, Typhon, 2021, p. 19.

116. A. FRAISSE, «René Maran », dans Hommage à René Maran, Paris, Présence Africaine, 1965, p. 271-276.

117. M. MBOUGAR SARR, « Préface », op. cit., p. 19. 
118. R. MARAN, « Préface », op. cit., p. 11.

119. R. MARAN, Un homme pareil aux autres, op. cit., p. 11.

120. R. MARAN, Le Cour serré, op. cit., p. 11.

121. "J'ai poussé la conscience objective jusqu'à y supprimer des réflexions que l'on aurait pu m'attribuer. » R. MARAN, « Préface », op. cit., p. 9

122. Ibid., p. 13

123. R. MARAN, « Au pied du mur », op. cit.

124. R. MARAN, Un homme pareil aux autres, op. cit., p. 133-134

125. D'après la transcription d'une lettre en date du 17 octobre 1948 adressée à Mercer Cook communiquée par Roger Little au sein de l'équipe René Maran de l'ITEM.

126. R. MARAN, Un homme pareil aux autres, op. cit., p. 11

127. N. Y. KISUDIKI, « Négritude et philosophie », art. cit., p. 1

128. R. MARAN, lettre à Marcel Martinet, 10 novembre 1929. Une lettre signalée et partagée par Harry Symes.

\section{RÉSUMÉS}

Les lettres adressées à Maurice Barrès au cours des années précédant la parution de Batouala, véritable roman nègre entre 1917 et 1920 offrent un éclairage sur l'intention et la mise en scène auctoriale de René Maran. Il semble en effet que l'écriture obéisse chez lui à un impératif ontologique, à savoir démontrer que «les nègres » sont des hommes comme les autres. L'auteur d'Un homme pareil aux autres se met ainsi en scène comme un exemple et un ambassadeur de la «cause » des nègres en tant que ceux-ci seraient des "êtres perfectibles ». La lecture de ces lettres permet d'apprécier le sens de son «métier d'écrivain français» dans la «lutte antiraciste » qu'il a pu mener au sein de la Ligue universelle de défense de la race nègre (LUDRN). Cet engagement peut se comprendre à l'aune d'une négritude en tant qu'elle est une «pratique sans théorie " (Roger Toumson) élaborée en fonction des «questions que pose la présence des Nègres au monde » et de « sa perception par l'Autre et par soi-même » (Romuald Fonkoua).

In the letters addressed to Maurice Barrès the years preceding Batouala, a true black novel publication, between 1917 and 1920, topics of Rene Maran authorial intention and exposure are highlighted. As a matter of fact, it seems writing may have been an ontological imperative, namely the demonstration that "negroes" are men like the others. The author of Un homme pareil aux autres stages himself as a prime example and ambassador of the black civil rights advancement because they are so-called "perfectible beings". Reading these letters allows us to assess the meaning of his "French writer profession", the "antiracist fight" he has been able to take part in The universal league for the defense of the negro race. It is in the light of negritude that his involvement could make sense being "a practice without a theory" built upon the "questions raised by the negro presence in the world" and "his perception by others and his selfperception". 
INDEX

Mots-clés : intention auctoriale, assimilation, mouvement abolitionniste, racisme, négritude

Keywords : Assimilation, abolitionnist movement, racism, negritude

\section{AUTEUR}

\section{XAVIER LUCE}

XAVIER LUCE est doctorant à l'Université Sorbonne au sein du Centre international d'études francophones (CIEF) et du Centre d'études de la langue et des littératures françaises (CELLF). Sa thèse, sous la direction de Romuald Fonkoua, porte sur Maryse Condé et sa critique. Il coordonne avec Claire Riffard au sein de l'Institut des textes et manuscrits modernes (ITEM) le séminaire René Maran. 